angiography was the gold standard for diagnosis of coronary artery disease, but to have invasive examination, only used in a small fraction of patients. Clinical diagnosis in most patients still need to rely on non-invasive examination. Coronary artery calcium deposition within the intima is a sign of atherosclerosis. CAC was associated with the presence and extent of coronary atherosclerosis.

Methods 280 symptomatic patients (mean age $60 \pm 5$; range 35 76 years) received coronary angiography and chest CT scan and their image were qualified. CT image shows left main and at least proximal and middle part of anterior descending was considered acceptable CT scans, punctate calcification as mild, segmental calcification as moderate and diffuse as severe. OCA was used to measure the stenosis within three vessels. Completely normal coronary artery was defined as no stenosis more than 30\%. Stenosis less than $50 \%$ was considered non-obstructive, while more than $50 \%$ stenosis as obstructive coronary artery disease.

Results There were no difference in patient with or without obstructive stenosis on demographic characteristics. Of all the 280 patients, 62 confirmed by coronary angiography with normal coronary artery, 218 patients in contrast with the narrow, including 59 non-obstructive, 159 of obstructive stenosis (96 with moderate CAC, 48 with severe CAC and 15 without). 62 patients with normal coronary angiography were not calcified. The sensitivity to predict normal coronary artery was $81.2 \%$, specificity was $100 \%$. Incidence and severity of CAC were associated with the severity of CAD.

Conclusions Symptomatic patients without CAC on common chest CT scan may have less possibility of CAD. Such patients may not need excessive coronary angiography. CAC on CT scan may predict multivessel disease before invasive coronary angiography.

\section{GW23-e0091 VALUE OF USING CT TO DETECT CORONARY CALCIFICATION ON DIAGNOSIS OF CORONARY ARTERY DISEASE}

doi:10.1136/heartjnl-2012-302920j.19
Sun Ling, Sun Ling. Board of health, Liaoning Fushun, Liaoning, China

Objectives Using the spiral CT to scan coronary calcification, with qualitative and semi-quantitative method, to predict the presence and extent of coronary artery disease. Early diagnosis of coronary artery disease has been an important clinical issue. Coronary 